\title{
Preliminary Evidence for a Compromised T-Cell Compartment in Maltreated Children with Depression and Posttraumatic Stress Disorder
}

\author{
Hannes Bielas ${ }^{a}$, b, e Andreas Jud ${ }^{b}$ e Ulrich Lips $^{b} \quad$ Janine Reichenbach ${ }^{c}$ \\ Iris Wieser ${ }^{a}$ Markus A. Landolta, d \\ ${ }^{a}$ Department of Psychosomatics and Psychiatry, ${ }^{b}$ Child Protection Team and ${ }^{\mathrm{C} D i v i s i o n}$ of Immunology, University \\ Children's Hospital Zurich, and d Department of Child and Adolescent Health Psychology, Institute of Psychology,

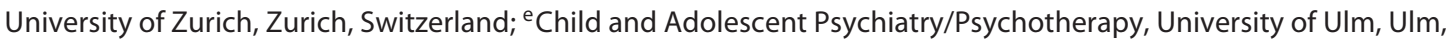 \\ Germany
}

\section{Key Words}

Adverse childhood experiences - Child maltreatment .

Depression - Posttraumatic stress disorder - Recent thymic emigrant cells $\cdot T$ cells

\begin{abstract}
Objective: Adverse childhood experiences, such as maltreatment, and affective disorders are associated with a proinflammatory state and/or variably compromised counts in lymphocyte subsets in adults. Animal models of social stress indicate that recent thymic emigrant cells (RTE), which maintain the T-cell compartment, are affected. Methods: In this study, we examined the association between lymphocyte subsets, and depression and posttraumatic stress disorder (PTSD) among 16 maltreated children (aged 6-17 years) 1-3 years after the intervention by the Child Protection Team and among 14 healthy age-matched controls. The participants completed psychological assessment and had blood drawn for fluorescent-activated cell sorting analysis. Results: Among maltreated children and adolescents, depression was associated with lower counts of RTEs and T-helper cells after controlling for age. We found additional trends and large effect sizes with regard to the percentages of these cells, as well as for related lymphocyte subsets. Similar ef-
\end{abstract}

fects were found for PTSD, i.e. lower counts of naïve T cells, which was also supported by a trend for their percentage. Compared to controls, maltreated participants with a clinical level of depression had decreased percentages of RTEs, with a similar trend for PTSD. Conclusion: Limited by the nature of a pilot study and the small sample size, these preliminary findings of a compromised T-cell compartment related to psychiatric symptoms in maltreated children and adolescents need to be further studied; particularly the role of RTEs needs further evaluation.

(c) 2015 S. Karger AG, Basel

\section{Introduction}

Adverse childhood experiences (ACEs) such as maltreatment are associated with long-term consequences for a victim's health $[1,2]$, including a dysregulated immune system $[3,4]$ and psychiatric disorders [5]. Additionally, psychopathology is considered to convey the effects of maltreatment on the immune system in later life [6]. As a result, depression [7] and posttraumatic stress disorder (PTSD) [8] have been related to up-regulated proinflammatory markers in adults with a history of child maltreatment. This was also found in 12-year-old chil-

\section{KARGER 125}

() 2015 S. Karger AG, Base

$1021-7401 / 15 / 0225-0303 \$ 39.50 / 0$

E-Mail karger@karger.com

www.karger.com/nim
Hannes Bielas, MD

Department of Psychosomatics and Psychiatry

University Children's Hospital Zurich

Steinwiesstrasse 75, CH-8032 Zurich (Switzerland)

E-Mail hannes.bielas@ kispi.uzh.ch 
dren with a history of maltreatment and depression [9]. Similarly, increased T-cell activation, which is mirrored by human leukocyte antigen (HLA)-DR expression, has recently been associated with maltreatment [10], but the role of comorbid psychopathology at a young age remains to be studied with regard to lymphocyte subsets.

Studies in adult patients with PTSD show mixed results with regard to $\mathrm{T}$-cell activation status and immune cell counts, i.e. the relationship between symptom severity and increased T-cell activation [8], or to associations between PTSD and decreased counts of lymphocytes, T cells [11] and naïve T lymphocytes [12]. The number of recent thymic emigrant cells (RTEs) in the T-cell compartment [13] has been found to be persistently reduced in mice showing PTSD-like behavior due to social stress $[14,15]$. However, early changes in the number and percentage of RTEs have not yet been evaluated in humans with respect to PTSD. The present paper provides preliminary data from such an examination in the aftermath of child maltreatment.

Based on the results obtained from studies in mammals, we expected reduced RTE counts and percentages in maltreated children with psychiatric diagnoses compared to healthy controls. From previous findings of increased proinflammatory markers, we hypothesized elevated counts and percentages of respective cells in the case of maltreatment that are reduced by the effect of a concomitant psychopathology such as PTSD and depression [16]. Hence, counts and percentages of the affected cell types were expected to inversely correlate with symptom severity, i.e. a compromised T-cell compartment was expected in maltreated children. Moreover, given the overlapping symptoms of depression and PTSD, we tried to determine whether the relationship between RTEs and depression accounts for the severity of comorbid PTSD and vice versa. Groups were controlled for the distribution of age [17], gender and socioeconomic status (SES) to avoid potential confounders [18].

\section{Materials and Methods}

\section{Study Cohort}

As part of a larger follow-up study by the Child Protection Team (CPT) at the University Children's Hospital Zurich [19], the present study included 31 participants (aged 7-16 years) who responded to questionnaires for the assessment of psychiatric symptoms and agreed to blood tests performed 1-3 years after the initial intervention by the CPT. Study groups consisted of 16 maltreated children and adolescents and 15 controls, who were matched for age, gender and nationality. The CPT applies definitions of child maltreatment that are based on widely accepted guidelines [20]. Cases were categorized as physical/sexual/psychological maltreatment or neglect applying exclusion criteria, for example the Munchausen syndrome by proxy or unsubstantiated cases. The exclusion criteria have previously been detailed elsewhere $[10,21]$. We also excluded 1 participant from the control group who reported clinical symptoms of depression beyond the cutoff value (see Psychopathology section). Participants and their primary caregivers consented to a face-to-face interview with a psychologist at the same hospital and to provide a blood sample for fluorescent-activated cell sorting (FACS) analysis. Children with inflammatory diseases or severe illnesses were excluded and, upon examinations of their health status, no clinically symptomatic viral or bacterial infections were reported at the time of FACS analysis. Written informed consent was obtained, and all procedures were approved by the local ethics committee.

\section{Lymphocyte Analysis}

FACS analyses of lymphocyte subpopulations were performed according to standard protocols by whole blood analysis (7-color approach, FACS-Diva software; Becton, Dickinson, Franklin Lakes, N.J., USA) for total lymphocytes (CD45+), B cells (CD19+/ CD45+), natural killer (NK) cells (CD3-CD16+CD56+/CD45+), monocytes (CD14+/CD45+) and total $\mathrm{T}$ cells (CD3+/CD45+). The CD3+ T-cell compartment was analyzed for T-helper (Th) cells (CD3+CD4+/CD45+), naïve $\mathrm{T}$ cells (CD4+CD45RA+/ CD3+), cytotoxic T cells (CD3+CD8+/CD45+), RTEs (CD31+ $\mathrm{CD} 45 \mathrm{RA}+/ \mathrm{CD} 4+)$ and their respective ratios. Moreover, the activation status of $\mathrm{T}$ cells was analyzed by HLA-DR expression, i.e. activated Th cells (CD4+HLA-DR+/CD3+) and cytotoxic T cells (CD8+HLA-DR+/CD3+). In subsequent analyses, sample sizes differed for several lymphocyte subsets due to limited availability of blood in some participants.

\section{Psychopathology}

PTSD was assessed using the University of California at Los Angeles PTSD Reaction Index, which displays good-to-excellent internal consistency and reliability across age ranges, sex and racial/ethnic groups in children aged $>6$ years [22]. The German version, comprising 32 items, which had previously displayed good internal consistency in a Swiss national sample (Cronbach's $\alpha=$ 0.86) [23] was applied in our study. Internal consistency among maltreated children and adolescents was good for all subscales (measuring intrusions, avoidance and hyperarousal) with a Cronbach's a between 0.84 and 0.93 , and excellent for the overall score (Cronbach's $\alpha=0.94$ ). The total PTSD severity score was computed by summing the score of all symptom items.

The severity of depressive symptoms was assessed by means of the Child Depression Inventory (CDI) [24], using the German version [25]. This 26-item questionnaire showed high validity, and a cutoff score was set at CDI $\geq 12$ (sensitivity $=91.7 \%$ and specificity $=81.9 \%$ ) according to the Youden Index [26].

\section{Control Variables}

In addition to age, gender and nationality (Swiss vs. non-Swiss), SES were assessed based on information on maternal education and current paternal employment. Both aspects were rated on a scale ranging from 1 to 6 , with 1 being the highest education and the highest employment status. Summed subscores represent the SES, ranging from the highest status of 2 to the lowest status of 12 . 
Table 1. Demographic characteristics of the study groups

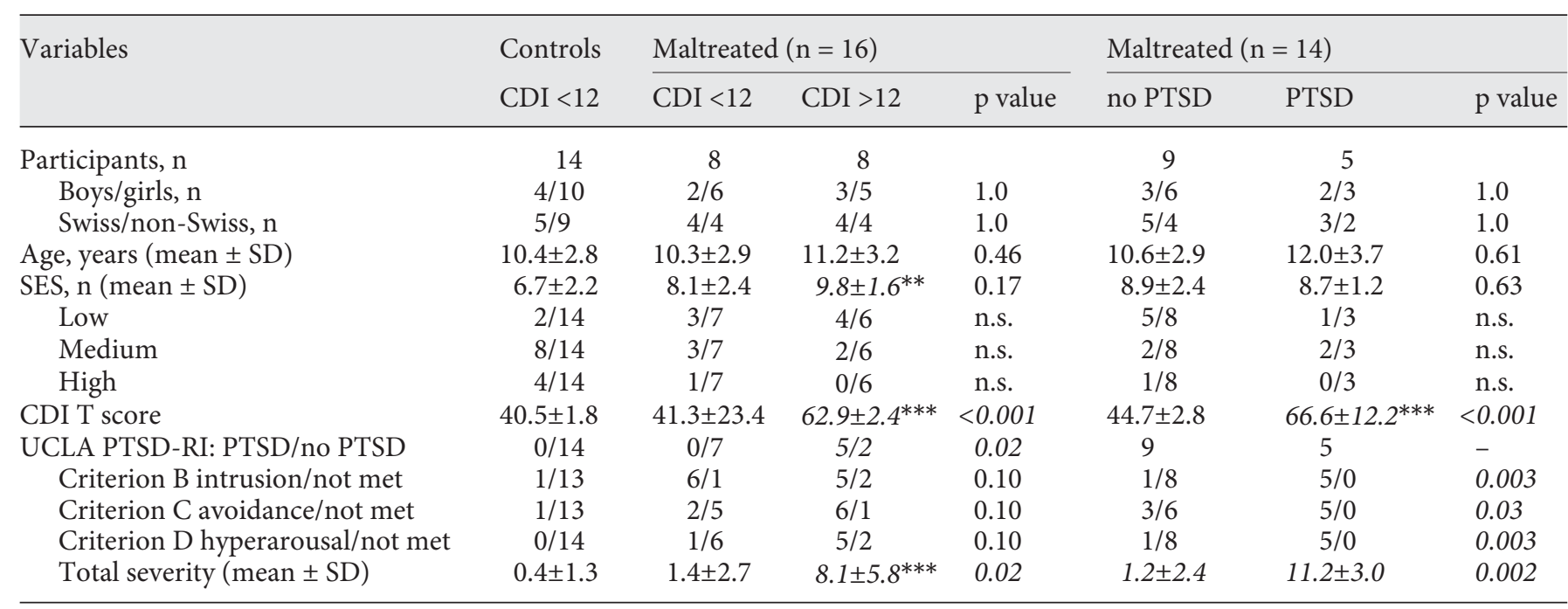

$\mathrm{RI}=$ Reaction Index. $\chi^{2}$ tests using Fisher's exact test and the Mann-Whitney U test were applied. Significant differences compared to controls and significant $\mathrm{p}$ values for comparison among maltreated participants are displayed in italics: ${ }^{* * *} \mathrm{p}<0.001,{ }^{* *} \mathrm{p}<0.01$; $\mathrm{p}$ value for overall group comparison.

To facilitate interpretation, scores were first reversed so that higher scores represented higher SES. Second, scores were assigned to one of three categories: low (2-5), medium (6-9) or high (10-12) high SES [27].

\section{Statistical Analyses}

Data were analyzed using SPSS for Windows, version 20 (SPSS Inc., Chicago, Ill., USA). All statistical tests were two sided with a predefined significance level of $\mathrm{p}<0.05 . \chi^{2}$ and Mann-Whitney $\mathrm{U}$ tests were used as appropriate to compare frequencies of aberrant cell counts and sociodemographic variables between groups of maltreated children with versus without depression, or PTSD diagnosis and controls. Cohen's $d$ effect sizes were computed to compare mean values of lymphocyte subsets between these groups [28]. Using analysis of covariance (ANCOVA), diagnostic categories were compared with regard to lymphocyte counts and percentages, thereby controlling for age as a covariate [29]. Normal distribution was assessed via Kolmogorov-Smirnov tests, and relationships between symptom severity and counts of lymphocyte subsets were assessed via partial correlation and multiple regression analyses, controlling for age accordingly.

\section{Results}

\section{Characteristics of the Participants}

All participants displayed counts of total lymphocytes within the respective age ranges $(\geq 10$ th and $\leq 90$ th percentiles). As seen in table 1, the groups studied did not differ versus controls with regard to sociodemographic confounders, such as gender, nationality or age; only SES

Psychopathology and Maltreatment

Compromises T Cells was higher among controls. SES did not differ between maltreated children with and without psychopathology.

Table 1 further shows that half of the 16 maltreated children and adolescents reported symptom severity scores above the cutoff for depression (mean $=9.3, \mathrm{SD}=$ 3.3 , range $=4.6-15.7$ in the whole sample). Five of the participants $(33 \%)$ met the diagnostic criteria for PTSD, while 2 of the 16 maltreated participants did not complete the PTSD questionnaire. No comparison between controls and maltreated children and adolescents with regard to depression and PTSD could be made, as these parameters served as exclusion criteria. However, there was a relevant overlap in self-reported symptoms of both diagnoses (table 1). Symptom severity of depression was higher in participants with PTSD. Participants with CDI scores above the cutoff also reported higher scores of PTSD severity in (sub)scales and were more likely to suffer from all DSM-IV PTSD criteria (table 1). According to the respective Cramer's $\varphi$ values, CDI scores above the cutoff were highly associated with a diagnosis of self-reported intrusions $(0.58, \mathrm{p}<0.05)$, avoidance $(0.58, \mathrm{p}<$ $0.05)$ and hyperarousal $(0.74, \mathrm{p}<0.01)$.

\section{Effect of Psychopathology on the Reduction in RTEs in Maltreated Participants}

As shown in table 2, there was a significant effect of maltreatment combined with depression on percentages of RTEs after controlling for the effect of age, $\mathrm{F}(2,26)=$ 
Table 2. Comparison of lymphocyte subsets (cells/nl) between maltreated children with and without depression, and controls (means \pm SD)

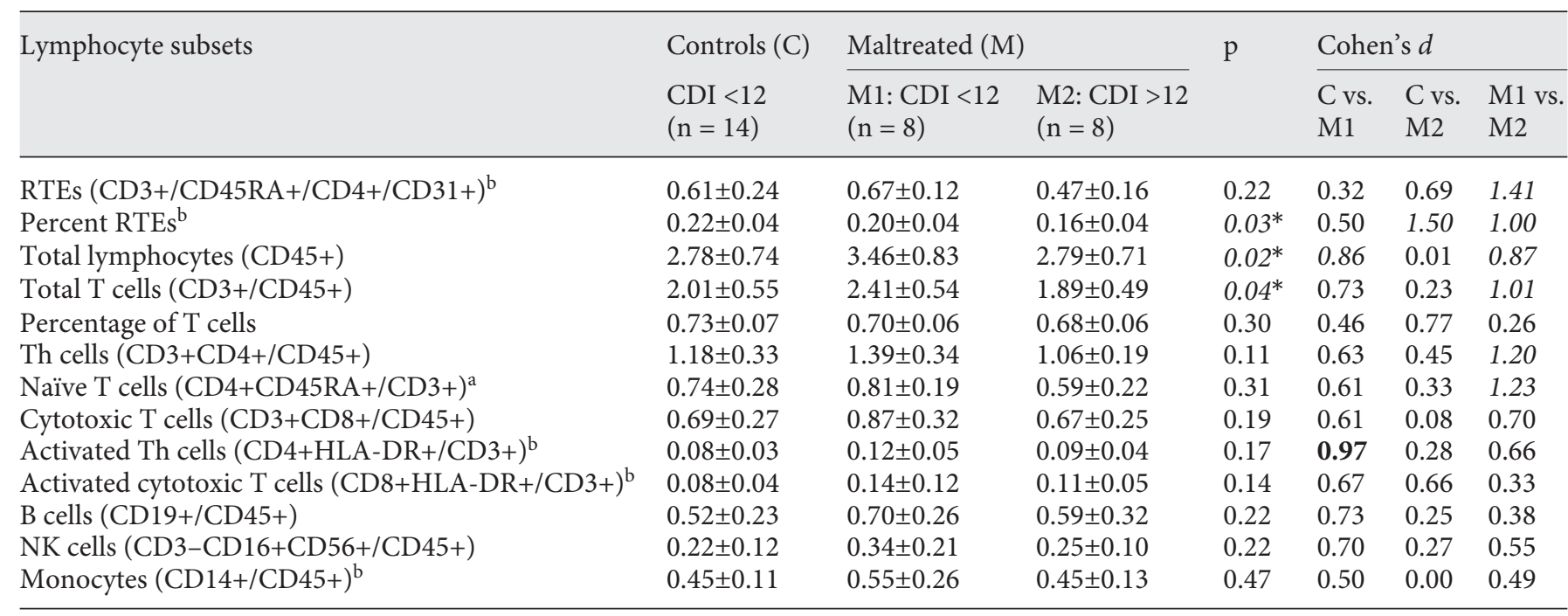

Analysis of covariance with 'age' as covariate and the depression cutoff score as between-subject factor (ANCOVA with p value for overall group comparison), estimating the effect size via Cohen's $d$ based on means \pm SD for the comparison of maltreated without depression (M1) vs. controls (C); maltreated with depression (M2) vs. C, as well as M1 vs. M2. Significant effects for cell counts were evaluated comparing correspondent percentages with respect to total lymphocyte counts. Large effect sizes (Cohen's $d>0.80)$ and significant differences ( $\mathrm{p}$ value) between groups $\left({ }^{*} \mathrm{p}<0.05\right)$ are displayed in italics. Sample sizes were restricted to ${ }^{\mathrm{a}}$ controls $(\mathrm{n}=11)$; maltreated $(n=6)$, and maltreated and depressed $(n=6)$, or ${ }^{b}$ maltreated $(n=7)$ and maltreated and depressed $(n=7)$.

Fig. 1. Depression after childhood maltreatment is associated with lower RTE counts. Box plots display RTE counts in children $1-3$ years after maltreatment with $(\mathrm{n}=7)$ and without depressive disorder $(\mathrm{n}=7)$, and in controls $(\mathrm{n}=12)$, quantified with FACS analysis, compared via one-way ANOVA. Means \pm SEM. ${ }^{* *} \mathrm{p}<0.01$ maltreated children with vs. without depression (effect size: Cohen's $d=1.41$ ). The difference between maltreated children with depression vs. controls was nonsignificant but showed a trend (effect size: Cohen's $d=$ $0.69 ; \mathrm{p}=0.43$ ).

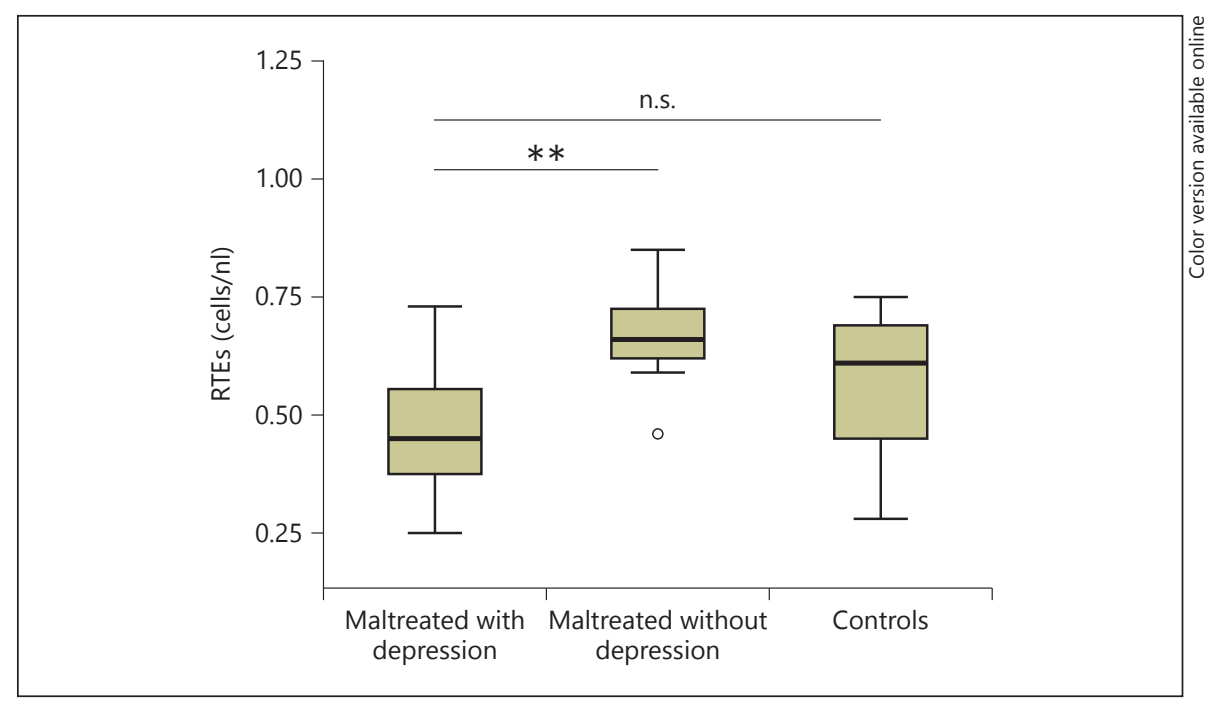

$4.16, \mathrm{p}<0.05$. Planned contrasts revealed that experience of maltreatment combined with current depression significantly decreased RTE percentages $(\mathrm{p}<0.01)$, whereas maltreatment alone did not $(\mathrm{p}=0.43$; fig. 1$)$. This was also reflected by RTE counts when effect sizes and Cohen's $d$ were compared between groups. Similarly, a marginal effect of maltreatment combined with PTSD resulting in reduced RTE percentages after controlling for the effect of age was noted, $\mathrm{F}(2,22)=2.80, \mathrm{p}=0.08$ (table 3 ). Planned contrasts revealed that experience of maltreatment combined with current depression significantly decreased RTE percentages $(\mathrm{p}<0.05)$, whereas maltreatment alone did not ( $p=0.33)$. Again, this was reflected by large effect sizes when mean RTE counts were compared. When as- 
Table 3. Comparison of lymphocyte subsets (cells/nl) between maltreated children with and without PTSD, and controls (means \pm SD)

\begin{tabular}{|c|c|c|c|c|c|c|c|}
\hline Lymphocyte subsets & $\begin{array}{l}\text { Controls }(\mathrm{C}) \\
\text { no PTSD } \\
(\mathrm{n}=14)\end{array}$ & \multicolumn{2}{|l|}{ Maltreated (M) } & $\mathrm{p}$ & \multicolumn{3}{|c|}{ Cohen's $d$} \\
\hline RTEs (CD3+/CD45RA+/CD4+/CD31+) $)^{\mathrm{b}}$ & $0.61 \pm 0.24$ & $0.65 \pm 0.13$ & $0.43 \pm 0.19$ & 0.44 & 0.21 & 0.83 & 1.35 \\
\hline Percent RTEs ${ }^{\mathrm{b}}$ & $0.22 \pm 0.05$ & $0.20 \pm 0.03$ & $0.16 \pm 0.05$ & $0.08\left(^{*}\right)$ & 0.49 & 1.2 & 0.97 \\
\hline Total lymphocytes (CD45+) & $2.78 \pm 0.74$ & $3.18 \pm 0.80$ & $2.68 \pm 0.70$ & 0.16 & 0.52 & 0.14 & 0.67 \\
\hline Naïve T cells $(\mathrm{CD} 4+\mathrm{CD} 45 \mathrm{RA}+/ \mathrm{CD} 3+)^{\mathrm{b}}$ & $0.74 \pm 0.28$ & $0.80 \pm 0.18$ & $0.52 \pm 0.21$ & 0.34 & & & 1.65 \\
\hline Cytotoxic T cells (CD3+CD8+/CD45+) & $0.69 \pm 0.27$ & $0.79 \pm 0.32$ & $0.59 \pm 0.12$ & 0.40 & 0.34 & 0.48 & 0.83 \\
\hline Activated Th cells $(\mathrm{CD} 4+\mathrm{HLA}-\mathrm{DR}+/ \mathrm{CD} 3+)^{\mathrm{b}}$ & $0.08 \pm 0.03$ & $0.11 \pm 0.05$ & $0.09 \pm 0.05$ & 0.35 & 0.73 & 0.24 & 0.49 \\
\hline Activated cytotoxic T cells (CD8+HLA-DR+/CD3+) ${ }^{\mathrm{b}}$ & $0.08 \pm 0.04$ & $0.14 \pm 0.12$ & $0.09 \pm 0.04$ & 0.18 & 0.67 & 0.25 & 0.56 \\
\hline $\mathrm{B}$ cells $(\mathrm{CD} 19+/ \mathrm{CD} 45+)$ & $0.52 \pm 0.23$ & $0.60 \pm 0.25$ & $0.67 \pm 0.37$ & 0.16 & 0.33 & 0.49 & 0.22 \\
\hline
\end{tabular}

Analysis of covariance with 'age' as covariate and the PTSD cutoff score as between-subject factor (ANCOVA with p value for overall group comparison); estimating the effect size via Cohen's $d$ based on means \pm SD for the comparison of maltreated without depression (M1) vs. controls (C); maltreated with depression (M2) vs. C, as well as M1 vs. M2. Significant effects for cell counts were evaluated comparing correspondent percentages with respect to total lymphocyte counts. Large effect sizes (Cohen's $d>0.80)$ and significant differences ( $\mathrm{p}$ value) between groups [trends $\left({ }^{*}\right) \mathrm{p}<0.1$ ] are displayed in italics. Sample sizes were restricted to ${ }^{\mathrm{a}}$ controls $(\mathrm{n}=11) ;{ }^{\mathrm{b}}$ maltreated $(n=6)$, and maltreated and depressed $(n=6)$, or ${ }^{c}$ maltreated $(n=7)$.

sessing the effect of depression and PTSD, RTE counts were mainly influenced by the covariate age $[\mathrm{F}(1,24)=$ $17.15, \mathrm{p}<0.001$, and $\mathrm{F}(2,22)=14.30, \mathrm{p}=0.001]$. In contrast, we found no relationship between the covariate age and the percentage of RTEs using ANCOVA.

\section{Effect of Depression on the Associations between}

Maltreatment and Mature T Cells

There was a significant effect of maltreatment on total lymphocyte counts after controlling for the effect of age, $\mathrm{F}(2,26)=4.40, \mathrm{p}<0.05$ (table 2). Planned contrasts revealed that maltreatment significantly increased lymphocyte counts $(p=0.007)$, whereas for the combination of maltreatment with depression this effect was diminished $(\mathrm{p}=0.43)$. Similarly, we found a significant effect of maltreatment on $\mathrm{T}$-cell counts after controlling for the effect of age, $\mathrm{F}(2,26)=3.50, \mathrm{p}<0.05$. Planned contrasts revealed that maltreatment significantly increased $\mathrm{T}$-cell counts compared to controls ( $p=0.02$ ), whereas the effect was diminished in the maltreatment group with depression $(\mathrm{p}=0.16)$. According to Cohen's $d$ (table 2), these differences in cell counts were accompanied by large effect sizes when maltreated participants with and without depressive symptom scores above the cutoff were compared.

Psychopathology and Maltreatment

Compromises T Cells
There were no group differences for any other lymphocyte subsets (ANCOVA), but the covariate age was significantly related to total lymphocyte counts: $\mathrm{F}(1,26)=$ 33.31, $\mathrm{p}<0.001$; B cells: $\mathrm{F}(1,26)=11.11, \mathrm{p}<0.01$; T cells: $\mathrm{F}(1,26)=34.02, \mathrm{p}<0.001$; Th cells: $\mathrm{F}(1,26)=9.80, \mathrm{p}<$ 0.01 ; activated Th cells: $\mathrm{F}(1,24)=4.36, \mathrm{p}<0.05$; cytotoxic T cells: $\mathrm{F}(1,26)=18.14, \mathrm{p}<0.001$, and naïve T cells: $\mathrm{F}(1,24)=17.15, \mathrm{p}<0.001$. In contrast, we found no relation between the covariate age and cell counts of NK cells, monocytes and cytotoxic T cells.

\section{Effect of PTSD on Mature T-Cell Counts of Maltreated Children}

Table 3 shows that Cohen's $d$ effect sizes were similar to those of depression, when lymphocyte counts of maltreated children and adolescents with and without PTSD were compared. However, we found no difference between groups with regard to mature T-cell subsets in ANCOVA. The covariate age was significantly related to total lymphocyte counts: $\mathrm{F}(2,24)=26.98, \mathrm{p}<0.001$; B cells: $\mathrm{F}(2,24)=12.0, \mathrm{p}<0.01$; $\mathrm{T}$ cells: $\mathrm{F}(2,24)=27.03$, $\mathrm{p}<$ 0.001 ; Th cells: $\mathrm{F}(2,24)=8.57, \mathrm{p}<0.01$, and cytotoxic $\mathrm{T}$ cells: $\mathrm{F}(2,24)=14.24, \mathrm{p}=0.001$. In contrast, we found no relationship between the covariate age and NK cell, monocyte and activated T-cell counts. 
Associations of Symptom Severity of PTSD and

Depression with RTEs

Spearman's $\rho$ coefficients were found to correlate inversely with symptom severity of the two diagnoses, the CDI $(\mathrm{n}=28, \mathrm{r}=-0.51, \mathrm{p}<0.01)$ and PTSD $(\mathrm{n}=26, \mathrm{r}=$ $-0.53, \mathrm{p}<0.01)$ in the whole sample. In the maltreated children, the results of regression analyses indicated that the combination of age and CDI explained $56.5 \%$ of the variance $\left[R^{2}=0.56, \mathrm{~F}(2,11)=9.45, \mathrm{p}<0.01\right]$. It was found that the CDI score significantly correlated with RTE counts $(r=-0.66, p<0.01)$, as did age $(r=-0.52, p<0.05)$. With regard to RTE percentages, on the other hand, only the CDI score was found to be a significant predictor $\left[R^{2}=0.38, \mathrm{~F}(1,12)=8.85, \mathrm{p}<0.05\right]$, correlating with the percentage of RTEs $(r=-0.65, p<0.01)$, whereas age did $\operatorname{not}(\mathrm{r}=0.18, \mathrm{p}=0.26)$.

PTSD severity was also found to predict RTE counts $\left[R^{2}=0.68, \mathrm{~F}(1,12)=24.86, \mathrm{p}<0.001\right]$. This was reflected by significant correlations between the total PTSD score and RTE counts $[\mathrm{r}(9)=-0.79, \mathrm{p}<0.01]$ and percentages $[\mathrm{r}(9)=-0.71, \mathrm{p}<0.05]$ via partial correlation analyses, controlling for age as a covariate. Similarly, naïve T cells correlated with the total PTSD score $[\mathrm{r}(9)=-0.83$, $\mathrm{p}<$ $0.05]$, which was also found to be a trend for $\mathrm{T}$-cell counts $[\mathrm{r}(11)=-0.52, \mathrm{p}<0.07]$, Th cells $[\mathrm{r}(11)=-0.54, \mathrm{p}=0.06]$, but not for total lymphocytes, B cells, monocytes, cytotoxic $\mathrm{T}$ cells and $\mathrm{T}$-cell subsets expressing activation markers.

\section{Discussion}

Confirming our hypothesis drawn from experimental data on social stress in mammals $[14,15]$, the percentages of RTEs were lower in maltreated children with selfreported PTSD levels compared with matched controls and maltreated children without the diagnosis. We found that self-reported depression was also associated with reduced RTE percentages, which is supported by large effect sizes when the group means of RTE counts and percentages for maltreated children with versus without depression or PTSD are compared. Our results are indirectly confirmed by a recent study showing that the association between a proinflammatory marker, C-reactive protein, and the combination of depression and maltreatment among school-aged children was not found for maltreatment or depression alone [9]. Thus, depression or PTSD may be factors driving the impaired maintenance of the T-cell compartment in the aftermath of ACEs such as maltreatment.
This hypothesis is supported by further analyses of the present data. The increase in total T-cell and total lymphocyte counts in maltreated participants with no psychiatric diagnosis appears to level off in cases of parallel depression. This is mirrored by large effect sizes for the comparison of group means throughout the data and the parallel trend for the counts of HLA-DR-expressing activated T cells [10]. In line with a meta-analysis showing an association between the decrease in the percentage of $\mathrm{T}$ cells and depression [16], the discount effect in our data is driven by linear correlations between counts and percentages of RTEs and the symptom severity of depression or PTSD among maltreated children and adolescents. The underlying mechanism may be an increased turnover of these activated cells under proinflammatory conditions reflected by increased C-reactive protein levels in maltreated children with depression [9]. Studies ignoring ACEs such as maltreatment may therefore fail to detect the association of adolescent depression and altered cellular immunity [30].

As hypothesized from the association of the two diagnoses even as early as in adolescence [31], PTSD severity no longer correlates with RTEs when the effect of depression is accounted for, and vice versa. Moreover, participants reported symptom severity above the cutoff for depression more frequently than for PTSD, which is a common finding in traumatized adolescents [18]. The subsequent imbalance of the small sample sizes in the groups studied may explain the lower number of findings with regard to PTSD.

However, according to our data, an increased total PTSD score was inversely correlated with RTE or naïve T cell counts, which also included a negative trend with respect to $\mathrm{T}$ and Th cells. This impact on the entire T-cell compartment is consistent with previous research in adult PTSD patients showing fewer naïve T lymphocytes but increased memory T lymphocytes [12]. Similarly, an increased ratio of memory versus naïve $\mathrm{T}$ cells was found in adult women with PTSD in the aftermath of childhood sexual abuse [32]. We hypothesized that the lower counts of total lymphocytes and total T cells in adults with a past history of PTSD [11] result from an accelerated age-related reduction in RTEs in the presence of a proinflammatory state, triggered by the mutual effects of T-cell activation and PTSD severity [8]. In line with this hypothesis, depressed adults show a decrease availability of $\mathrm{T}$ cells, which has been related to an increased sensitivity to apoptosis [33]. Clinical implications of such a compromised T-cell compartment related to psychiatric morbidity [34] need to be examined in a larger study including also analyses of cytokine profiles, for example. 
The lack of comparative data from children and adolescents regarding the dysregulation of cellular immune responses following maltreatment and/or psychopathological disorders impairs further interpretation of our results. The latter are also limited by the small sample size and the character of this pilot study with its cross-sectional design, implicating the risk of selection biases and limited statistical power, especially for the assessment of potential confounders. Therefore, the combined effect of psychopathology and maltreatment can only be stated with caution, without generalizing the effect for the combination of depression or PTSD with other ACEs [10]. Notwithstanding this, the current study is the first to demonstrate a negative impact of depression and PTSD on RTEs among maltreated children controlled for age. These preliminary findings indicate that a long-term effect of psychiatric disorders after trauma in early life is associated with a compromised T-cell compartment. Future studies in larger samples including the assessment of cytokine profiles, for example, are warranted.

\section{Acknowledgments}

This study was funded by the 'Perspectives' foundation of Swiss Life, Zürich, and the Olga Mayenfisch Foundation, Zürich. J.R. was supported by the Gebert Rüf Stiftung, Program 'Rare Diseases - New Approaches'. The authors have no financial relationships with the organizations that sponsored the research. We would like to thank Dr. L. Molinari and S. Bollmann, M.Sc., for helpful comments on the statistical analyses.

\section{Disclosure Statement}

The authors have no conflict of interest to disclose.

\section{References}

1 Felitti VJ, Anda RF, Nordenberg D, Williamson DF, Spitz AM, Edwards V, et al: Relationship of childhood abuse and household dysfunction to many of the leading causes of death in adults. The Adverse Childhood Experiences (ACE) Study. Am J Prev Med 1998; 14:245-258.

- 2 Gilbert R, Widom CS, Browne K, Fergusson D, Webb E, Janson S: Burden and consequences of child maltreatment in high-income countries. Lancet 2009;373:68-81.

-3 Dube SR, Fairweather D, Pearson WS, Felitti VJ, Anda RF, Croft JB: Cumulative childhood stress and autoimmune diseases in adults. Psychosom Med 2009;71:243-250.

-4 Fagundes CP, Glaser R, Kiecolt-Glaser JK: Stressful early life experiences and immune dysregulation across the lifespan. Brain Behav Immun 2013;27:8-12.

$\checkmark 5$ Horwitz AV, Widom CS, McLaughlin J, White HR: The impact of childhood abuse and neglect on adult mental health: a prospective study. J Health Soc Behav 2001;42:184201.

6 Kiecolt-Glaser JK, McGuire L, Robles TF, Glaser R: Psychoneuroimmunology: psychological influences on immune function and health. J Consult Clin Psychol 2002;70:537547.

7 Danese A, Moffitt TE, Pariante CM, Ambler A, Poulton R, Caspi A: Elevated inflammation levels in depressed adults with a history of childhood maltreatment. Arch Gen Psychiatry 2008;65:409-415.

8 Lemieux A, Coe CL, Carnes M: Symptom severity predicts degree of $\mathrm{T}$ cell activation in adult women following childhood maltreatment. Brain Behav Immun 2008;22:9941003.
-9 Danese A, Caspi A, Williams B, Ambler A, Sugden K, Mika J, et al: Biological embedding of stress through inflammation processes in childhood. Mol Psychiatry 2011;16:244-246.

10 Bielas H, Jud A, Lips U, Reichenbach J, Landolt MA: Increased number of activated T cells in lymphocyte subsets of maltreated children: data from a pilot study. J Psychosom Res 2012;73:313-318.

11 Kawamura N, Kim Y, Asukai N: Suppression of cellular immunity in men with a past history of posttraumatic stress disorder. Am J Psychiatry 2001;158:484-486.

12 Sommershof A, Aichinger H, Engler H, Adenauer $\mathrm{H}$, Catani C, Boneberg EM, et al: Substantial reduction of naive and regulatory $\mathrm{T}$ cells following traumatic stress. Brain Behav Immun 2009;23:1117-1124.

13 Fink PJ: The biology of recent thymic emigrants. Annu Rev Immunol 2013;31:31-50.

14 Engler H, Bailey MT, Engler A, Sheridan JF: Effects of repeated social stress on leukocyte distribution in bone marrow, peripheral blood and spleen. J Neuroimmunol 2004;148: 106-115.

15 Engler H, Stefanski V: Social stress and T cell maturation in male rats: transient and persistent alterations in thymic function. Psychoneuroendocrinology 2003;28:951-969.

16 Zorrilla EP, Luborsky L, McKay JR, Rosenthal R, Houldin A, Tax A, et al: The relationship of depression and stressors to immunological assays: a meta-analytic review. Brain Behav Immun 2001;15:199-226.

17 Schatorjé EJH, Gemen EFA, Driessen GJA, Leuvenink J, van Hout RWNM, de Vries E: Paediatric reference values for the peripheral T cell compartment. Scand J Immunol 2012; 75:436-444.
18 Gerson R, Rappaport N: Traumatic stress and posttraumatic stress disorder in youth: recent research findings on clinical impact, assessment, and treatment. J Adolesc Health 2013; 52:137-143.

19 Jud A, Lips U, Landolt MA: Characteristics associated with maltreatment types in children referred to a hospital protection team. Eur J Pediatr 2010;169:173-180.

20 Leeb RT, Paulozzi L, Melanson C, Simon T, Arias I: Child Maltreatment Surveillance: Uniform Definitions for Public Health and Recommended Data Elements, Version 1.0. Atlanta, Centers for Disease Control and Prevention, National Center for Injury Prevention and Control, 2008, http://www.cdc.gov/violence prevention/pdf/CM_Surveillance-a.pdf.

-21 Jud A, Lips U, Landolt MA: Methodological challenges in following up patients of a hospital child protection team: is there a recruitment bias? Child Adolesc Psychiatry Ment Health 2010;4:27.

22 Steinberg AM, Brymer MJ, Kim S, Briggs EC, Ippen CG, Ostrowski SA, et al: Psychometric properties of the UCLA PTSD reaction index: part I. J Trauma Stress 2013;26:1-9.

23 Landolt MA, Schnyder U, Maier T, Schoenbucher V, Mohler-Kuo M: Trauma exposure and posttraumatic stress disorder in adolescents: a national survey in Switzerland. J Trauma Stress 2013;26:209-216.

24 Kovacs M: The Children's Depression, Inventory (CDI). Psychopharmacol Bull 1985;21: 995-998.

25 Stiensmeierpelster J, Schurmann M, Urhahne D: Inventory of Depression in Children and Adolescents (Dikj) - usability in primary grades. Z Entwicklungspsychol Padagog Psychol 1991;23:171-176. 
26 Frühe B, Allgaier A-K, Pietsch K, SchulteKörne G: Depression screening in pediatric patients - a comparison of the concurrent validity of the German version of the Children's Depression Inventory, the German Depression Test for Children, and the new Children's Depression Screener (in German). Z Kinder Jugendpsychiatr Psychother 2012;40:161-169.

-27 Largo RH, Pfister D, Molinari L, Kundu S, Lipp A, Duc G: Significance of prenatal, perinatal and postnatal factors in the development of AGA preterm infants at five to seven years. Dev Med Child Neurol 1989;31:440-456.
28 Cohen J: A power primer. Psychol Bull 1992; 112:155-159.

29 Houston EG, Boursalian TE, Fink PJ: Homeostatic signals do not drive post-thymic T cell maturation. Cell Immunol 2012;274:39-45.

30 Targum SD, Clarkson LL, Magac-Harris K, Marshall LE, Skwerer RG: Measurement of cortisol and lymphocyte subpopulations in depressed and conduct-disordered adolescents. J Affect Disord 1990;18:91-96.

31 Allwood MA, Dyl J, Hunt JI, Spirito A: Comorbidity and service utilization among psychiatrically hospitalized adolescents with posttraumatic stress disorder. J Psychol Trauma 2008;7:104-121.
32 Wilson RS, Boyle PA, Levine SR, Yu L, Anagnos SE, Buchman AS, et al: Emotional neglect in childhood and cerebral infarction in older age. Neurology 2012;79:1534-1539.

33 Miller AH: Depression and immunity: a role for T cells? Brain Behav Immun 2010;24:1-8.

34 Ironson G, Wynings C, Schneiderman N, Baum A, Rodriguez M, Greenwood D, et al: Posttraumatic stress symptoms, intrusive thoughts, loss, and immune function after Hurricane Andrew. Psychosom Med 1997;59: 128-141. 